\title{
COUNTEREXAMPLE TO THE MORSE-SARD THEOREM IN THE CASE OF INFINITE- DIMENSIONAL MANIFOLDS
}

\section{IVAN KUPKA}

1. Introduction. The classical Morse-Sard [1], [2] theorem in the case of a function says the following: Let $f: \Omega \rightarrow R$ be a $C^{n}$-function, where $\Omega$ is an open set in $R^{n}$. Then, the set of critical values of $f$ is of measure zero. Recall that $c \in R$ is a critical value of $f$ if $f^{-1}(c)$ contains a point $x \in \Omega$ where $d f_{x}=0$.

As is well known, the theorem is false if we replace the assumption " $f \in C^{n}$ " by " $f \in C^{k}$," where $k<n[3]$. In case $\Omega$ is replaced by an open set in a Hilbert or Banach space $B$, it is easy to see that the theorem is false if either (i) $f$ is not required to be $C^{\infty}$, or (ii) $B$ is not separable. It is only too natural to ask whether the following generalization is true: Let $F: \Omega \rightarrow R$ be $C^{\infty}$, where $\Omega$ is an open set in a separable Hilbert space $H$. Then, the set of critical points for $F$ forms a set of measure zero.

As we shall show in the following, this generalization is false. We construct a $C^{\infty}$ function $f: H \rightarrow R$, with $H=l^{2}$, such that the singular set is the Cantor set $\Sigma \subset H$ formed by the sequences $\left(\alpha_{1}, \alpha_{2} / 2, \alpha_{3} / 3, \cdots, \alpha_{n} / n, \cdots\right)$ where, for all $n, \alpha_{n}=0$ or 1 . Our example also shows that the Morse-Sard theorem does not hold, in the generalized form, even if we require that the singular set be compact. The set of critical points for $F$ will be the interval $[0,1]$.

2. Let $H^{*}$ be the dual of $H$. A base of $H^{*}$ is formed by the linear functions $e_{1}, e_{2}, \cdots, e_{n}, \cdots$, where

$$
e_{n}(x)=x_{n} \text { for } x=\left(x_{1}, x_{2}, \cdots, x_{k}, \cdots\right) \text {. }
$$

For any $k$, let $\Sigma_{k}\left(H^{*}\right)$ be the space of all $k$-linear symmetric continuous functions on $H . \Sigma_{k}\left(H^{*}\right)$ is also a Hilbert space, with norm \|\|$_{k}$. Clearly, $\Sigma_{1}\left(H^{*}\right)=H^{*}$.

To each $e_{n}$ and each $k=1,2,3, \cdots$, there is canonically attached an element $\left(e_{n}\right)^{k}$ in $\Sigma_{k}\left(H^{*}\right)$ defined by

$$
\left(e_{n}\right)^{k}\left(x^{1}, x^{2}, \cdots, x^{k}\right)=e_{n}\left(x^{1}\right) e_{n}\left(x^{2}\right) \cdots e_{n}\left(x^{k}\right)
$$

for any points $x^{1}, x^{2}, \cdots, x^{k}$ in $H$. Note that, for any $k,\left\|\left(e_{n}\right)^{k}\right\|_{k}=1$.

To each $x \in H$, we associate a continuous linear operator $z(x)$ : $\Sigma_{k}\left(H^{*}\right) \rightarrow \Sigma_{k-1}\left(H^{*}\right)$ for all $k \geqq 2$ by:

Received by the editors September 17, 1963. 
For any $a \in \Sigma_{k}\left(H^{*}\right)$, let

$$
z(x)(a)\left(x^{1}, x^{2}, \cdots, x^{k-1}\right)=a\left(x, x^{1}, x^{2}, \cdots, x^{k-1}\right) .
$$

The norm of $z(x)$ is $\|x\|$, for $\|z(x) a\|_{k-1} \leqq\|x\|\|a\|_{k}$ and $z(x)\left(e_{n}\right)^{k}$ $=x_{n}\left(e_{n}\right)^{k-1}$.

Now, let $g: \Omega \rightarrow R$ be a function defined on the open set $\Omega \subset H$. We say that $g$ is of class $C^{\infty}$ if there exists a sequence of continuous mappings: $L^{k}: x \in \Omega \rightarrow L_{x}^{k} \in \Sigma_{k}\left(H^{*}\right)$ such that, for any $\epsilon>0$ and any integer $N>0$, there exists a $\delta>0$ such that, if $x \in \Omega$ and $y \in H$, with $\|y\|<\delta$, then

$$
\left|g(x+y)-g(x)-L_{x}^{k}(y)\right| \leqq \epsilon\|y\|
$$

and

$$
\left\|L_{x+y}^{k}-L_{x}^{k}-z(y) L_{x}^{k+1}\right\|<\epsilon\|y\|,
$$

for $k=1,2,3, \cdots, N$. The sequence $L_{x}^{1}, L_{x}^{2}, \cdots$ is then unique, and $L_{x}^{k}$ is called the $k$ th derivative of $g$ at $x$, and is written $d^{k} g_{x}$.

3. We first introduce a $C^{\infty}$-function $\lambda: R \rightarrow R$ with the following properties: (i) $\lambda$ is an increasing $C^{\infty}$-function, (ii) $-1 \leqq \lambda(t) \leqq 2$ for all $t,-\infty<t<\infty$, with $\lambda(0)=0, \lambda(1)=1$, (iii) $0 \leqq \lambda^{\prime}(t) \leqq 2$, and $\lambda^{\prime}(t)=0$ only for $t=0$ or $t=1$, (iv) when $|t| \rightarrow \infty, \lambda^{(k)}(t) \rightarrow 0$ for each $k=1,2, \cdots$, and if $t \rightarrow \infty, \lambda(t) \rightarrow 2$, and if $t \rightarrow-\infty, \lambda(t) \rightarrow-1$. Note that there are many such functions $\lambda$. Having chosen one, let $M_{k}$ be the maximum of $\left|\lambda^{(k)}(t)\right|$ for $t \in R$.

Now, define the function $F: H \rightarrow R$ as follows: For $x \in H$ $x=\left(x_{1}, x_{2}, \cdots\right)$, set

$$
F(x)=\sum_{n=1}^{\infty} \frac{\lambda\left(n x_{n}\right)}{2^{n}} .
$$

This series is clearly absolutely convergent for all $x \in H$. We now show that the function so defined is in $C^{\infty}$.

The sequence of mappings $L^{k}: x \in H \rightarrow L_{x}^{k} \in \Sigma_{k}\left(H^{*}\right)$ is given by

$$
L_{x}^{k}=\sum_{n=1}^{\infty} \frac{n^{k} \lambda^{(k)}\left(n x_{n}\right)}{2^{n}}\left(e_{n}\right)^{k} .
$$

This series converges and defines a member of $\Sigma_{k}\left(H^{*}\right)$ for each $x \in H$ since the series of norms \|\|$_{k}$ of its terms, i.e.,

$$
\sum_{n=1}^{\infty} \frac{n^{k}\left|\lambda^{(k)}\left(n x_{n}\right)\right|}{2^{n}}
$$

is dominated by the series $\sum_{n=1}^{\infty} n^{k} M_{k} / 2^{n}$, which converges. We next 
show that the map $x \rightarrow L_{x}^{k}$ is continuous. Take any $r \in H$. Then,

$$
L_{x+r}^{k}-L_{x}^{k}=\sum_{n=1}^{\infty} \frac{n^{k}\left\{\lambda^{(k)}\left(n x_{n}+n r_{n}\right)-\lambda^{(k)}\left(n x_{n}\right)\right\}}{2^{n}}\left(e_{n}\right)^{k} .
$$

By Taylor's formula,

$$
\lambda^{(k)}\left(n x_{n}+n r_{n}\right)-\lambda^{(k)}\left(n x_{n}\right)=n r_{n} \int_{0}^{1} \lambda^{(k+1)}\left(n x_{n}+t n r_{n}\right) d t,
$$

so that

$$
\left|\lambda^{(k)}\left(n x_{n}+n r_{n}\right)-\lambda^{(k)}\left(n x_{n}\right)\right| \leqq n r_{n} M_{k+1} .
$$

This leads to the estimate

$$
\left\|L_{x+r}^{k}-L_{x}^{k}\right\|_{k} \leqq \sum_{n=1}^{\infty} \frac{n^{k+1} M_{k+1}}{2^{n}}\left|r_{n}\right| \leqq\|r\| \sigma_{k+1}
$$

where $\sigma_{k}=\sum_{n-1}^{\infty} n^{k} M_{k} / 2^{n}$.

We next calculate $L_{x+r}^{k}-L_{x}^{k}-z(r) L_{x}^{k+1}$, which is easily seen to be

$$
\sum_{n=1}^{\infty} \frac{n^{k}}{2^{n}}\left\{\lambda^{(k)}\left(n x_{n}+n r_{n}\right)-\lambda^{(k)}\left(n x_{n}\right)-n r_{n} \lambda^{(k+1)}\left(n x_{n}\right)\right\}\left(e_{n}\right)^{k}
$$

Another application of Taylor's formula to this yields the estimate $\left\|L_{x+r}^{\boldsymbol{k}}-L_{x}^{\boldsymbol{k}}-z(r) L_{x}^{\boldsymbol{k}+1}\right\| \leqq \sigma_{k+2}\|r\|^{2}$. It then follows that $F$ is of class $C^{\infty}$.

Let us now determine the critical points for $F$. We have

$$
d F_{x}=\sum_{n=1}^{\infty} \frac{n \lambda^{\prime}\left(n x_{n}\right)}{2^{n}} e_{n}
$$

since $\left(e_{n}\right)^{1}=e_{n}$. To have $d F_{x}$ identically zero, we need $\lambda^{\prime}\left(n x_{n}\right)=0$ for all $n=1,2, \cdots$. By assumption, $\lambda^{\prime}(t)=0$ only if $t=0$ or $t=1$. The point $x \in H$ must therefore obey: $x_{n}=0$ or $1 / n$, for every $n$. This identifies $x$ as a member of the set Cantor-type set $\Sigma \subset H$. The critical-point set for $F$ will be the set of points $F(x)$, for $x \in \Sigma$. Let $x=\left(\alpha_{1}, \alpha_{2} / 2\right.$, $\left.\alpha_{3} / 3, \alpha_{4} / 4, \cdots\right)$, where each $\alpha_{n}$ is either 0 or 1 . Then,

$$
F(x)=\sum_{1}^{\infty} \frac{\lambda\left(n x_{n}\right)}{2^{n}}=\sum_{n=1}^{\infty} \frac{\lambda\left(\alpha_{n}\right)}{2^{n}} .
$$

Since $\lambda(0)=0$ and $\lambda(1)=1$, we have $F(x)=\sum_{1}^{\infty} \alpha_{n} / 2^{n}$, so that the critical-point set for $F$ is the entire interval $[0,1]$, which is of measure 1 . We observe that the function $\lambda$ could have been chosen so that not only the first, but, indeed, all the derivatives of $F$ vanish on the set $\Sigma$. 


\title{
BIBLIOGRAPHY
}

1. A. P. Morse, The behavior of a function on its critical set, Ann. of Math. 40 (1939), 62-70.

2. Arthur Sard, The measure of the critical values of differentiable maps, Bull. Amer. Math. Soc. 48 (1942), 883-890.

3. H. Whitney, $A$ function not constant on a connected set of its critical points, Duke Math. J. 1 (1935), 514-517.

Instituto de Mathematica, Pura e Aplicada, Rio de Janeiro, Brazil

\section{THE DEGREE OF APPROXIMATION BY LINEAR OPERATORS}

\author{
PHILIP C. CURTIS, JR. ${ }^{1}$
}

I. Introduction. In 1959 Berman [1] observed the following: If $T$ denotes the unit circle, $C(T)$ the continuous functions on $T$, and $E_{n}(f)$ the error in the best approximation in the sup norm to $f$ by a trigonometric polynomial of order $n$, then there could not exist a sequence of linear operators $T_{n}$ mapping $C(T)$ into the trigonometric polynomials of order $n$, which satisfied

$$
\left\|f-T_{n} f\right\| \leqq K E_{n}(f)
$$

for some fixed constant $K$ and $f \in C(T)$. The reason for this is easy to see. Suppose (1) is satisfied for some sequence $\left\{T_{n}\right\}$ and all $f \in C(T)$. If $\Pi_{n}$ denotes the space of trigonometric polynomials of order $\leqq n$, then for $f \in \Pi_{n}, E_{n}(f)=0$. Hence, $T_{n} f=f$ or $T_{n}^{2}=T_{n}$. But by a theorem of Nikolaev [3, p. 494], $\left\|T_{n}\right\| \geqq K \log n$. Since $E_{n}(f) \rightarrow 0$, this is a contradiction. A similar observation holds for $L_{1}(T)$ where $E_{n}(f)$ is the best approximation by a trigonometric polynomial of order $n$ to $f$ in the $L_{1}$ norm.

In this note I would like to elaborate on this observation of Berman's and make some applications to Fourier series which appear to be new. Let $T_{n}$ be a sequence of bounded linear operators from $C(T)$ into $\Pi_{n}$. In place of $E_{n}(f)$ let $D_{n}(f)$ be any continuous mapping from $C(T)$ to the non-negative reals which vanishes on $\Pi_{n}$. In addition to the case $D_{n}(f)=E_{n}(f)$ we may take $D_{n}(f)=\left\|f-P_{n} f\right\|$ where $P_{n}$ is a bounded projection of $C(T)$ onto $\Pi_{n}$. Then, if $T_{n}^{2} \neq T_{n}$ for each $n$, it

Received by the editors February 20, 1964.

1 The research reported here was supported by TRW Space Technology Laboratories and by the National Science Foundation. 